\title{
Site of rewarding action of morphine in the mesolimbic system determined by intracranial electrical self-stimulation
}

\author{
LEO VAN WOLFSWINKEL and JAN M. VAN REE \\ Rudolf Magnus Institute for Pharmacology, Medical Faculty, University of Utrecht, Utrecht (The Netherlands)
}

(Accepted July 30th, 1985)

Key words: self-stimulation — threshold - morphine - ventral tegmental area - nucleus accumbens

\begin{abstract}
Systemic treatment with morphine has been shown to facilitate intracranial electrical self-stimulation reward elicited from the ventral tegmental area (VTA) as was determined using a response rate-insensitive threshold measurement. In the present experiment graded doses of morphine were microinjected into the mesolimbic system to determine the site of this morphine action. Morphine injected into the nucleus accumbens did not affect the threshold and response rate of self-stimulation by electrodes in the VTA, while relatively high doses of morphine injected into the VTA produced a long-lasting decrease of the threshold of self-stimulation by electrodes in the nucleus accumbens. It is concluded that morphine can facilitate self-stimulation when injected into the VTA, and that a concerted action of morphine on multiple brain sites may be involved in the interaction of the drug with brain reward.
\end{abstract}

Opiates are used for their analgesic properties, but there is also a widespread non-medical use, which may be related to the euphorigenic and rewarding properties of these drugs 6,15 . These rewarding effects are probably not identical to the analgesic properties; they differ e.g. in the development of tolerance, which occurs quite fast for analgesia and, if present at all, much slower for the rewarding effects. Moreover, different brain sites may be involved in the analgesic and the rewarding effects of opiates ${ }^{1}$. Thus, particularly for analgesia, sites within the central gray of the brainstem are important ${ }^{20}$, while the most likely brain sites for the rewarding action of opiates are within the mesolimbic dopaminergic system ${ }^{18}$. The cell bodies of this system are located in the ventral tegmental area (VTA), and its fibers run through the medial forebrain bundle to many forebrain regions, including the nucleus accumbens (NA), the prefrontal cortex and septal regions ${ }^{4}$. Using self-injection and place preference techniques, rewarding activities of opiates have been observed in the VTA $^{2,9,13,16 .}$

Self-stimulation studies have shown that direct electrical activation of a number of brain sites is re- warding ${ }^{12}$. These sites are mainly, but probably not exclusively, related to dopaminergic systems, and particularly to the mesolimbic dopaminergic system. Because of the similarities between self-stimulation and opiate reward we have investigated the interaction between opiates and brain stimulation reward in the mesolimbic system. In previous experiments we found that morphine, administered subcutaneously (s.c.) in rats in doses up to $5 \mathrm{mg} / \mathrm{kg}$, dose-dependently decreased the self-stimulation threshold determined in rats with electrodes in the VTA ${ }^{17}$. The present experiments were aimed at the site of this action of morphine in the mesolimbic system. Thus, morphine was administered locally into the NA in rats with an electrode in the VTA and into the VTA in rats with an electrode in the NA. Threshold for electrical self-stimulation was determined using a procedure which has been shown to be relatively insensitive to motor performance deficits induced by morphine ${ }^{17,21}$. It was found that morphine administration into the VTA increased self-stimulation reward, while injection of morphine into the NA was not effective.

Male Wistar rats (TNO, Zeist, The Netherlands)

Correspondence: J.M. van Ree, Rudolf Magnus Institute for Pharmacology, Medical Faculty, University of Utrecht, Vondellaan 6, 3521 GD Utrecht, The Netherlands. 
from our own breeding stock, weighing $200-220 \mathrm{~g}$ at the time of the operation, were stereotaxically implanted with electrodes made from twisted stainless steel wire of $200 \mu \mathrm{m}$ thickness, insulated except for the cross-section at the tip, and with a stainless steel guide cannula for microinjections. In one group (VTA stimulation group) the electrode was located in the VTA (coordinates: A 2.6, D 3.7, L 1.0, according to De Groot (see ref. 8)) and the cannula in the ipsilateral NA, which was inserted making an angle of $15^{\circ}$ with the sagittal plane (coordinates: A 2.6, D $0.4, \mathrm{~L} 1.5$ ). In the other group (NA stimulation group) the electrode was placed in the NA and the cannula in the ipsilateral VTA, using the same set of coordinates. After the operation the rats were housed in single transparent cages with free access to food and water. At least one week after the operation the self-stimulation training started. The experiments were done in an aluminum Skinner box with a Plexiglas front door (Campden Instruments, U.K., model 410 ). Two retractable levers were present, 6 $\mathrm{cm}$ above the stainless steel grid floor and $14 \mathrm{~cm}$ apart. Via a mercury swivel and a spring-shielded lead the electrode could be connected to the stimulator (type ST, Janssen, Beerse, Belgium), which pro- duced 0.5 -s trains of bipolar rectangular pulses with a frequency of $100 \mathrm{~Hz}$. Pulse duration and interval between the positive and negative pulse were $0.5 \mathrm{~ms}$. The maximal current was adjusted individually for each rat. A self-constructed, programmable input voltage-dependent constant current unit regulated the current to the electrodes in steps of $1 \%$ of the maximal current. The rats had to press the right-hand lever to obtain stimulation (stimulation lever). The current intensity was maximal at the beginning of the session, while after every response the current was decreased by $1 \%$. The current intensity could be reset to maximal by a response on the left hand lever (reset lever). Above the stimulation lever a white light was present which was turned off during the stimulus train. A white light above the reset lever was off after a reset response until responding on the stimulation lever was resumed. Details of the training procedure are described elsewhere ${ }^{17}$. The total number of responses on the stimulation lever during the 25-min session indicated total response rate. The threshold was expressed as the mean of the currents at which the reset lever was operated during the session. The rats received one session per day for 5 days a week. They were repeatedly tested with graded

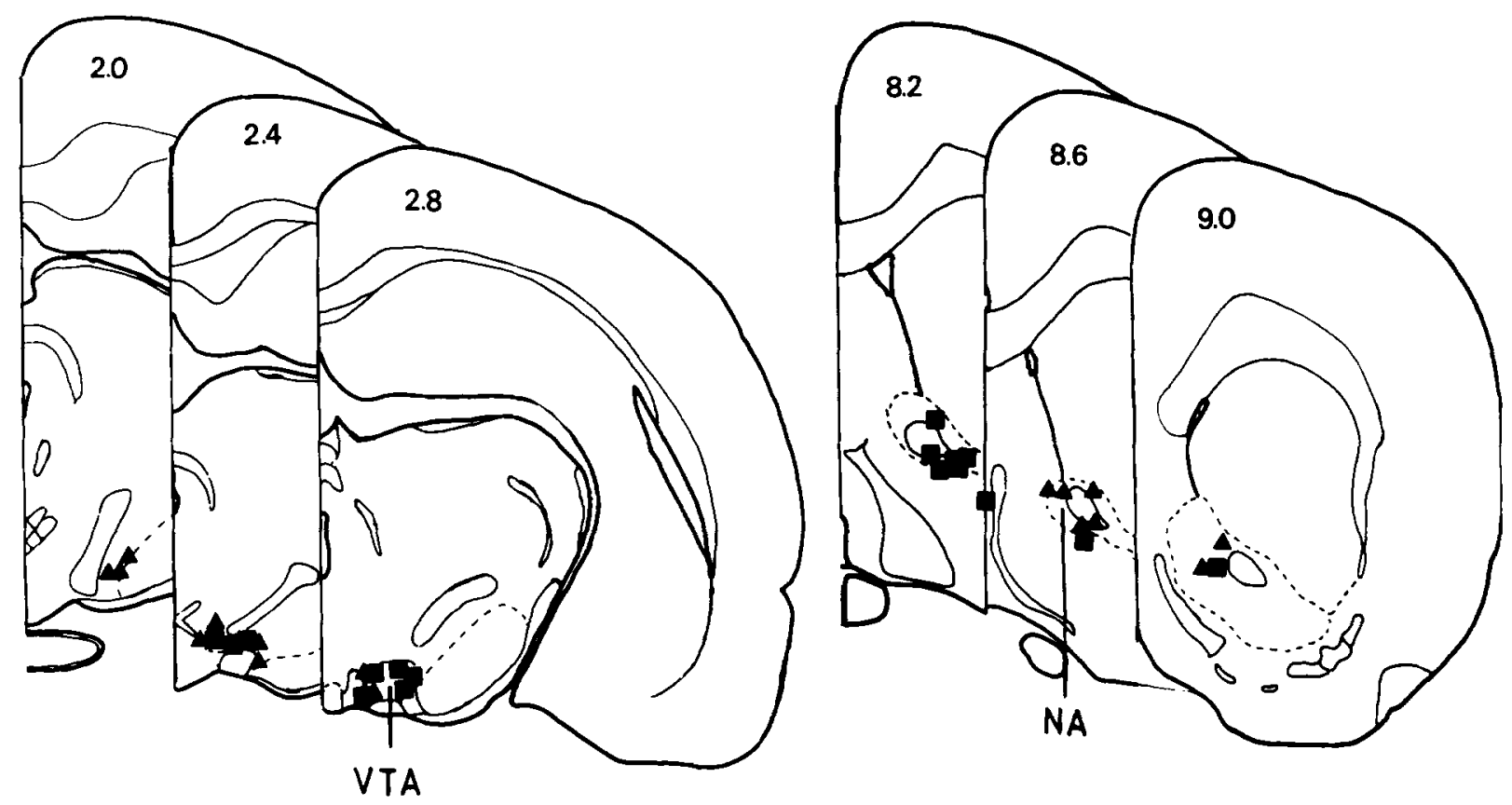

Fig. 1. Location of electrodes $(\boldsymbol{\Delta})$ and injection sites $(\mathbf{D})$ in the rats used in the experiments. Animals were equipped with electrodes in the VTA or in the NA and injection sites were in the NA or in the VTA, respectively. Sections were redrawn after Pellegrino et al. ${ }^{8}$. 


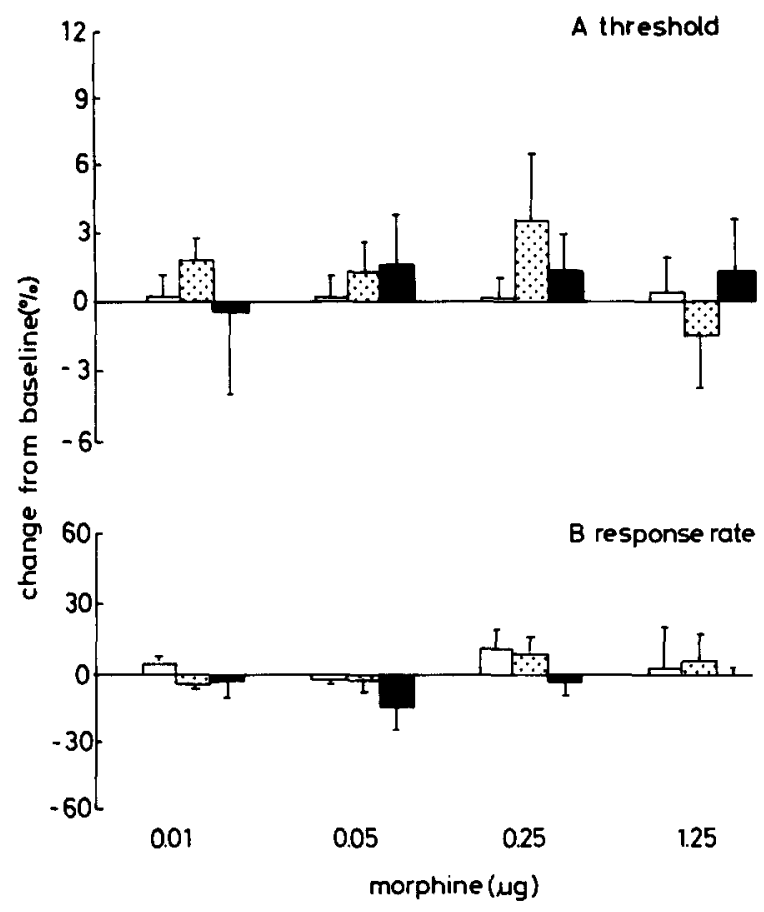

Fig. 2. Changes of self-stimulation threshold (A) and response rate (B) after injection of graded doses $(0.01-1.25 \mu \mathrm{g})$ of morphine in the NA of rats with electrodes in the VTA. Data are expressed as percentage of the performance in the session on the day (day 2) before active treatment (baseline session). Each dose level was tested in 6-10 rats. Open column, saline injection (day 1); dotted column, morphine treatment (day 3); closed column, no injection (day 4).

doses of morphine, administered in ascending order. On the 2 nd or the 3 rd day of the week saline $(1 \mu \mathrm{l})$ was injected $10 \mathrm{~min}$ before the session. Two days later the drug was injected, dissolved in $1 \mu \mathrm{l}$ of saline, via the cannula in the VTA or in the NA. The performance of the rats was expressed as the percentage of the performance on day 3 or day 4 , when no injection was given. Effects of treatment on response rate and threshold during the session were tested with analysis of variance for repeated measures, followed by Student's $t$-test for dependent variables. After the experiment the rats were decapitated, their brains quickly removed and stored in $4 \%$ formalin. The location of the tips of the electrodes and cannulas were verified in thionin-stained frozen sections of $100 \mu \mathrm{m}$ thickness.

The location of the electrodes and the cannulas in the experimental groups is shown in Fig. 1. All cannulas and electrodes were located within a narrow region in the VTA and in the NA. The rats readily learned to press the lever for self-stimulation. The rats with electrodes in the NA had a somewhat lower and more stable response rate as compared to those in which the VTA was stimulated. Response rates during the threshold procedure ranged from 28 to 67/min and from 6 to $29 / \mathrm{min}$ for the VTA and NA group, respectively. At maximal current (ranging from 50 to $220 \mu \mathrm{A}$ ), without active treatment, rates were $39-70 / \mathrm{min}$ and $19-40 / \mathrm{min}$, respectively. Training to stable performance took 4-6 weeks. Injections with saline into the NA or into the VTA did not change the threshold and response rate in any of the experimental weeks as compared to those on the noinjection day (Figs. 2 and 3). The absolute threshold currents in $\mu \mathrm{A}$ on the no-injection day (baseline) are shown in Table I. The variation of threshold current intensity over the whole experiment is less than $10 \%$. In some rats a slight gradual decrease in threshold over the sessions was present, whereas in others the threshold remained on the same level or increased somewhat. Therefore, the no-injection day that preceded the drug-test day was selected as reference day (baseline) to reduce the effect of this drift in individual rats. Injection of morphine $(0.01-1.25 \mu \mathrm{g})$ into the

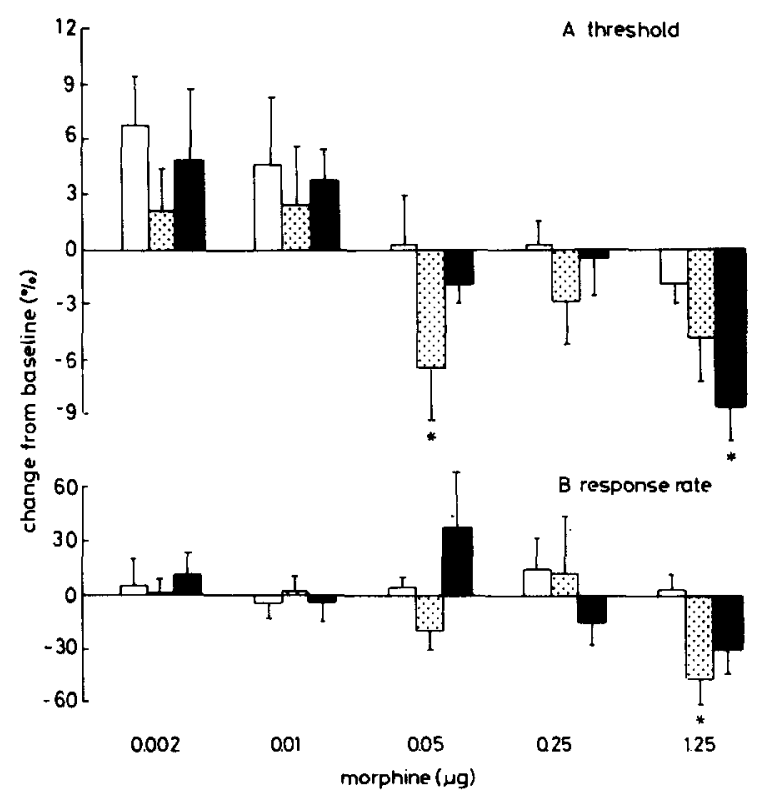

Fig. 3. Changes of self-stimulation threshold (A) and response rate (B) after injection of graded doses $(0.002-1.25 \mu \mathrm{g})$ of morphine in the VTA of rats with electrodes in the NA. For further details see legend to Fig. 2. ( ${ }^{*} P<0.05$, Student's $t$-test for paired samples.) 


\section{TABLE I}

Mean threshold current in $\mu \mathrm{A}$ ( \pm S.E.M.) in the sessions with out injection that preceded the injection of various doses of morphine in the NA or in the VTA in rats with an intracranial self-stimulation electrode in the VTA or the NA, respectively.

\begin{tabular}{llcclll} 
Injection & \multicolumn{5}{l}{ Dose of morphine $(\mu \mathrm{g})$} \\
site & 0.002 & 0.01 & 0.05 & 0.25 & 1.25 \\
NA & & $112 \pm 21$ & $112 \pm 21$ & $105 \pm 22$ & $100 \pm 26$ \\
VTA & $69 \pm 6$ & $64 \pm 4$ & $67 \pm 4$ & $67 \pm 7$ & $77 \pm 9$
\end{tabular}

NA had no significant effect on the threshold of rats with electrodes in the VTA (Fig. 2A, ANOVA for repeated measures, dose effects, $F_{3,21}=0.19$, n.s., dose by time interaction, $F_{9,46}=0.56$, n.s.). Also, response rate during the session was not affected by morphine treatment (Fig. 2B, dose effects, $F_{3,21}=$ 1.14 , n.s., dose by time interaction, $F_{9,46}=0.49$, n.s.). In an additional experiment also no effect of naloxone injections $(0.5 \mu \mathrm{g})$ into the NA was found in the rats with electrodes in the VTA. Injection of morphine $(0.002-1.25 \mu \mathrm{g})$ into the VTA changed the self-stimulation thresholds of rats with electrodes in the NA (Fig. 3A, ANOVA, dose effect: $F_{4,36}=4.42$, $P<0.01$, dose by session interaction, $F_{12,90}=1.96, P$ $<0.05$ ). The response rate during the session, however, was not significantly affected by morphine treatment (Fig. 3B, dose effect $F_{4,36}=1.26$, n.s., dose by session interaction $F_{12,90}=1.45$, n.s.). The change in threshold appeared to be caused by a decrease after $0.05 \mu \mathrm{g}$ of morphine $\left(t_{8}=2.35, P<0.05\right.$, Student's $t$-test for paired samples), and a decrease $24 \mathrm{~h}$ after $1.25 \mu \mathrm{g}\left(t_{7}=4.26, P<0.01\right)$, but also on the day when this latter dose of morphine was injected a tendency to a decrease was observed. Both threshold and response rate were returned to baseline when measured in the next session, which was performed 4 days after the morphine injection. In previous experiments using the present test procedure, we have found that s.c. injected morphine dose-dependently lowers the self-stimulation threshold of rats with an electrode in the VTA ${ }^{17}$. In rats with an electrode in the NA we observed in an additional experiment that a s.c. injection with $2.5 \mathrm{mg} / \mathrm{kg}$ morphine lowered the threshold to $87 \pm 2 \%$ of basal level $(P<0.05)$, while on the next day the threshold had returned to $99 \pm 2 \%$ of this level.
The present data show that administration of morphine into the VTA affects the threshold for selfstimulation by electrodes in the NA. This effect is comparable to that observed after s.c. administration of morphine. However, the effective dose for local injection into the VTA is quite high, which may suggest that the action of peripherally injected morphine on the threshold cannot be completely assigned to an action of morphine on the VTA. Thus, while this area seems to be important for morphine reward, probably the concerted action of morphine on a number of sites in the brain may be responsible for the change of self-stimulation threshold. The same holds for the analgesic action of opiates, in that the amount of opiates to obtain analgesia following local injection into the brain is much higher than the amount which is present after a peripheral injection of an analgesic dose of opioids, suggesting that multiple sites in the central nervous system contribute to the analgesic action of opiates $10,14,19$. The finding that the effect of $1.25 \mu \mathrm{g}$ of morphine on the threshold was even stronger after $24 \mathrm{~h}$ than just after the injection suggests that morphine is still present after one day, which may be due to the slow rate of biotransformation of morphine in the brain 5.10 . Morphine may have spread from the injection site, and the low concentration of this drug in a wider area may influence the threshold more than a high amount in the restricted area around the tip of the cannula ${ }^{3}$. But an effect that lasts longer than the presence of opiates in the brain cannot be ruled out. The lack of effect of morphine injected into the NA on the threshold of rats with electrodes in the VTA agrees with the observation showing that intra-accumbal opiate self-administration is hardly observed in drug-naive rats, but maintenance of self-administration in experienced rats has been reported ${ }^{7}$. Although electrical stimulation in the NA is rewarding and opioid binding sites have been found in this region ${ }^{11}$, opiates might not stimulate this region in a way comparable to electrical stimulation. Another possibility is that opiate injection into the NA activates the reward system via the VTA, and as VTA stimulation completely depolarizes the neuronal elements around the tip, the opioid stimulation of the NA does not add to the rewarding effect of electrical stimulation.

In conclusion, morphine injected into the VTA increases self-stimulation reward as determined with a 
response rate-insensitive threshold measurement and morphine injected into the NA is not effective. Thus, the site of rewarding action of morphine in the mesolimbic system may be in the VTA, which agrees with observations using other techniques to determine rewarding effects of opiates $2,9,16$. Although the VTA may be a sensitive site for morphine reward, in-

1 Bozarth, M.A. and Wise, R.A., Anatomically distinct opiate receptor fields mediate reward and physical dependence, Science, 224 (1984) 516-517.

2 Bozarth, M.A. and Wise, R.A., Intracranial self-administration of morphine into the ventral tegmental area in rats, Life Sci., 28 (1981) 551-555.

3 Broekkamp, C.L., Phillips, A.G. and Cools, A.R., Facilitation of self-stimulation behavior following intracerebral microinjections of opioids into the ventral tegmental area, Pharmacol. Biochem. Behav., 11 (1979) 289-295.

4 Lindvall, O. and Björklund, A., Anatomy of the dopaminergic neuron systems in the rat brain, $A d v$. Biochem. Psychopharmacol., 19 (1978) 1-24.

5 Mullis, K. B., Perry, D.C., Finn, A.M., Stafford, B. and Sadee, W., Morphine persistence in rat brain and serum after single doses, J. Pharmacol. Exp. Ther., 208 (1979) 228-231.

6 Nicholi, A.M., The nontherapeutic use of psychoactive drugs. A modern epidemic, N. Engl. J. Med., 308 (1984) 925-933.

7 Olds, M.E., Reinforcing effects of morphine in the nucleus accumbens, Brain Research, 237 (1982) 429-440.

8 Pellegrino, L.J., Pellegrino, A.S. and Cushman, A.J., A Stereotaxic Atlas of the Rat Brain, Plenum Press, New York, 1979.

9 Phillips, A.G. and LePaine, F.G., Reinforcing effects of morphine microinjections into the ventral tegmental area, Pharmacol. Biochem. Behav., 12 (1980) 965-986.

10 Plomp, G.J.J., Maes, R.A.A. and Van Ree, J.M., Disposition of morphine in rat brain: relationship to biological activity, J. Pharmacol. Exp. Ther., 217 (1981) 181-188.

11 Pollard, H., Llorens, C., Bonnet, J.J., Costentin, J. and Schwartz, J.C., Opiate receptors on mesolimbic dopaminergic neurons, Neurosci. Lett., 7 (1977) 295-299.

12 Prado-Alcala, R., Streather, A. and Wise, R.A., Brain stimulation reward and dopamine terminal fields. II. Septal teraction of this drug with other brain sites may contribute to the ultimate level of reward elicited by systemic administration of opiates.

This research was supported by a grant from the Ministry of Welfare, Health and Cultural Affairs, The Netherlands.

and cortical projections, Brain Research, 301 (1984) 209-219.

13 Stewart, J., Reinstatement of heroin and cocaine self-administration in the rat by intracerebral application of morphine in the ventral tegmental area, Pharmacol. Biochem. Behav., 20 (1984) 917-923.

14 Van Ree, J.M., Multiple brain sites involved in morphine antinociception, J. Pharm. Pharmacol., 29 (1977) 765-767.

15 Van Ree, J.M., Slangen, J.L. and De Wied, D., Intravenous self-administration of drugs in rats, J. Pharmacol. Exp. Ther., 204 (1978) 547-557.

16 Van Ree, J.M. and De Wied, D., Involvement of neurohypophyseal peptides in drug-mediated adaptive responses, Pharmacol. Biochem. Behav., 13, Suppl. 1 (1980) 257-263.

17 Van Wolfswinkel, L. and Van Ree, J.M., Effects of morphine and naloxone on ventral tegmental electrical selfstimulation. In F.C. Colpaert and J.L. Slangen (Eds.), Drug Discrimination: Applications in CNS Pharmacology, Elsevier, Amsterdam, 1982, pp. 391-397.

18 Wise, R.A. and Bozarth, M.A., Action of drugs of abuse on brain reward systems: an update with specific attention to opiates, Pharmacol. Biochem. Behav., 17 (1982) 239-243.

19 Yaksh, T.L. and Rudy, T.A., Analgesia mediated by a direct spinal action of narcotics, Science, 192 (1976) $1357-1358$.

20 Yaksh, T.L., Yeung, J.C. and Rudy, T.A., Systematic examination in the rat of brain sites sensitive to the direct application of morphine: observation of differential effects within the periaqueductal gray, Brain Research, 114 (1976) 83-103.

21 Zarevics, P. and Setler, P.E., Simultaneous rate-dependent and rate-independent assessment of intracranial selfstimulation: evidence for the direct involvement of dopamine in brain reinforcement mechanisms, Brain Research, 169 (1979) 499-512. 